\title{
Experimental and Computational Investigation of
}

\author{
Acetic Acid Deoxygenation over Oxophilic
}

\section{Molybdenum Carbide: Surface Chemistry and}

\section{Active Site Identity}

Joshua A. Schaidle ${ }^{*,+}$, Jeffrey Blackburn ${ }^{*}$, Carrie Farberow ${ }^{\dagger}$, Connor Nash $^{\dagger}$, K. Xerxes Steirer ${ }^{\#}$, Jared Clark ${ }^{\dagger}$, David Robichaud ${ }^{\prime}$, and Daniel A. Ruddy $y^{*}$

${ }^{\dagger}$ National Bioenergy Center, ${ }^{\ddagger}$ Chemistry and Nanoscience Center, and ${ }^{\#}$ Materials Science Center, National Renewable Energy Laboratory, Golden, Colorado 80401, United States

*Corresponding Author: Joshua.Schaidle@nrel.gov

\section{Contents}

i. Experimental Details - Mass Spectroscopy Deconvolution

ii. Supporting Figures

iii. Supporting Tables

iv. Supporting References 
i. Experimental Details - Mass Spectroscopy Deconvolution

Reactants and products were monitored during temperature programmed reaction (TPRxn) experiments with an online mass spectrometer (RGA 100, Stanford Research Systems). Table S1 displays the relative intensities for mass fragments of a given compound. As mass fragmentation patterns are known to be instrument specific, ${ }^{1-3}$ pure compound fragmentation patterns were collected using the online MS for all compounds except ethane and were incorporated into the deconvolution algorithm. For ethane, the mass fragmentation pattern was obtained from the NIST Chemistry WebBook database. ${ }^{4}$

The mass fragments in Table S1 were recorded as a function of reaction temperature and corrected for overlapping signals, or deconvoluted, according to a method adapted from Zhang, et al. $^{5}$ A system of linear equations was derived from mass conservation to solve for the corrected signals of each compound as a function of temperature and is expressed as

$$
\boldsymbol{R}=\boldsymbol{C} * \boldsymbol{M S}
$$

where $\boldsymbol{R}$ is a matrix of $N_{T}$ rows by $N_{i}$ columns representing the raw mass spectral data set. Each row of $\boldsymbol{R}$ is the mass spectrum at a given temperature $(T)$ and each column corresponds to a mass-to-charge ratio $(i) . \mathbf{C}$ is a $N_{T}$ by $N_{c}$ matrix of corrected signals for all of the $N_{c}$ compounds. $\boldsymbol{M S}$ is the $N_{c}$ by $N_{i}$ fragmentation pattern displayed in Table $\mathrm{S} 1$. The system of linear equations may be solved for $\boldsymbol{C}$ to obtain the deconvoluted signal for each $N_{c}$ compound as a function of temperature. As a result of the linear algebra operations, each $N_{c}$ compound signal in $C$ was normalized to an aggregate fragmentation pattern intensity, $F_{m, n o r m a l}$, which accounts for contributions to the compound signal from more than one mass-to-charge ratio. For example, $F_{m, \text { normal }}$ for hydrogen is equal to the normalized intensity of the primary mass fragment $\left(F_{m, n o r m a l}\right.$ $=100$ for $m / z=2$ ) whereas for acetaldehyde $F_{m \text {,normal }}$ is equal to 132.8 as a result of overlapping 
signals in the fragmentation pattern, and thus multiple contributions to the compound signal. The deconvolution method was completely dependent on the fragmentation pattern, $\boldsymbol{M S}$, and did not require any user input (e.g., primary mass fragment identification). The deconvoluted data were then corrected similarly to the method described by Ko, et al. for relative differences in ionization efficiency $\left(I_{x}\right)$, quadrupole transmission $\left(T_{m}\right)$, and electron multiplier gain $\left(G_{m}\right){ }^{6}$ The ionization efficiency is primarily dependent on the number of electrons per molecule expressed as

$$
I_{x}=0.6 * \frac{\left[\# \text { of } e^{-}\right]}{14}+0.4
$$

The gain of the electron multiplier is a function of ion mass and was calculated relative to carbon monoxide $(\mathrm{CO})$

$$
G_{m}=\sqrt{\frac{28}{M W}}
$$

In addition, the quadrupole transmission is also a function of ion mass and was approximated as

$$
T_{m}=\left\{\begin{array}{cc}
10^{(30-M W) / 155} & M W>30 \\
1 & M W<30
\end{array}\right\}
$$

The final correction factor $\left(C_{F}\right)$ is given by

$$
C_{F}=\frac{1}{100 * I_{x}} * \sum_{\text {mass fragment }} \frac{F_{m}}{G_{m} * T_{m}}
$$

where the summation is over all mass fragments for the compound and $F_{m}$ is the normalized fragmentation pattern intensity of the mass fragment. The final mass spectrum data is found by multiplying the deconvoluted data $(\boldsymbol{C})$ by the correction factors shown in Table S2. Primary mass fragments in Table S2 were identified based on the solution to Equation S1. The primary mass fragments were considered the major fragments contributing to the respective species signal in $\boldsymbol{C}$, 
but by no means were they considered the only contributing fragments, as the deconvoluted signals in $\boldsymbol{C}$ represent the complex fragmentation overlap in $\boldsymbol{M S}$.

Reactant conversion during TPRxn experiments was calculated using Equation S6:

$$
X_{r}=\left(1-\frac{I_{r}}{I_{r, i}}\right) \times 100
$$

where $X_{r}$ is the conversion of reactant $r$ (i.e., acetic acid, acetaldehyde, ethanol, or $\mathrm{H}_{2}$ ), $I_{r}$ is the normalized intensity of reactant $r$, and $I_{r, i}$ is the initial normalized intensity of reactant $r$ prior to the start of the TPRxn experiment. Reactant consumption rates, $R$, were calculated according to Equation S7:

$$
R=\frac{X_{r} \times F_{r, i}}{m_{\text {cat }} \times N_{\text {sites }}}
$$

where $F_{r, i}$ is the molar flow rate of reactant $r$ at the inlet of the reactor, $m_{\text {cat }}$ is the mass of catalyst loaded into the reactor, and $N_{\text {sites }}$ is the active site density (sites $/ \mathrm{g}_{\text {cat }}$ ) as determined by $\mathrm{H}_{2}$ chemisorption or $\mathrm{NH}_{3}$ temperature programmed desorption. 
ii. Supporting Figures

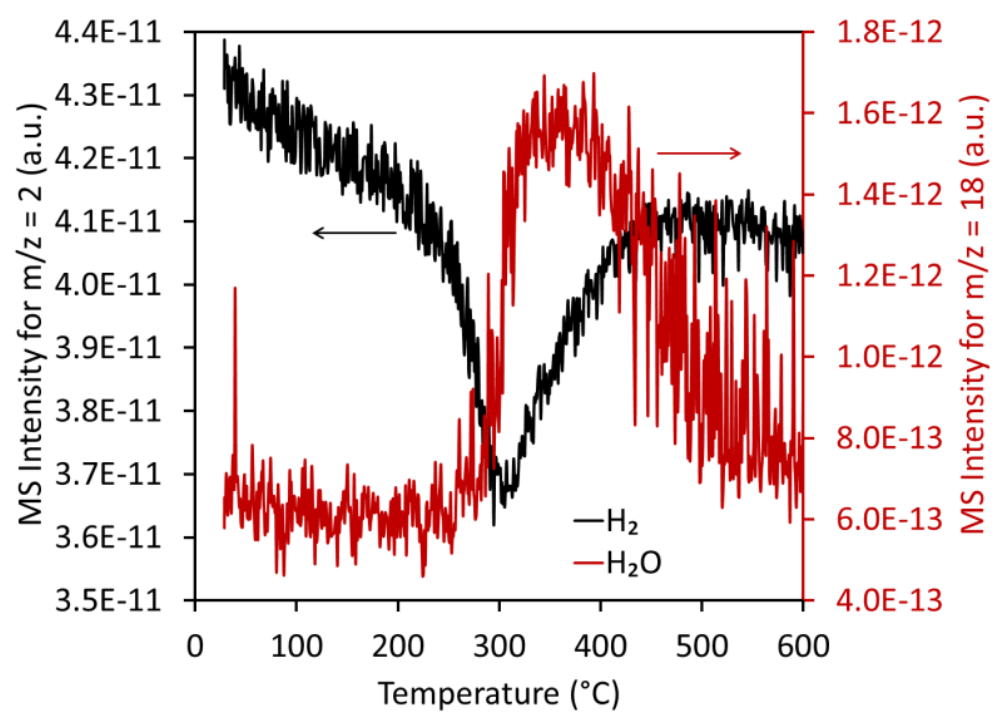

Figure S1. Consumption of $\mathrm{H}_{2}$ and production of $\mathrm{H}_{2} \mathrm{O}$ during a temperature programmed reduction of $\mathrm{Mo}_{2} \mathrm{C}$. 

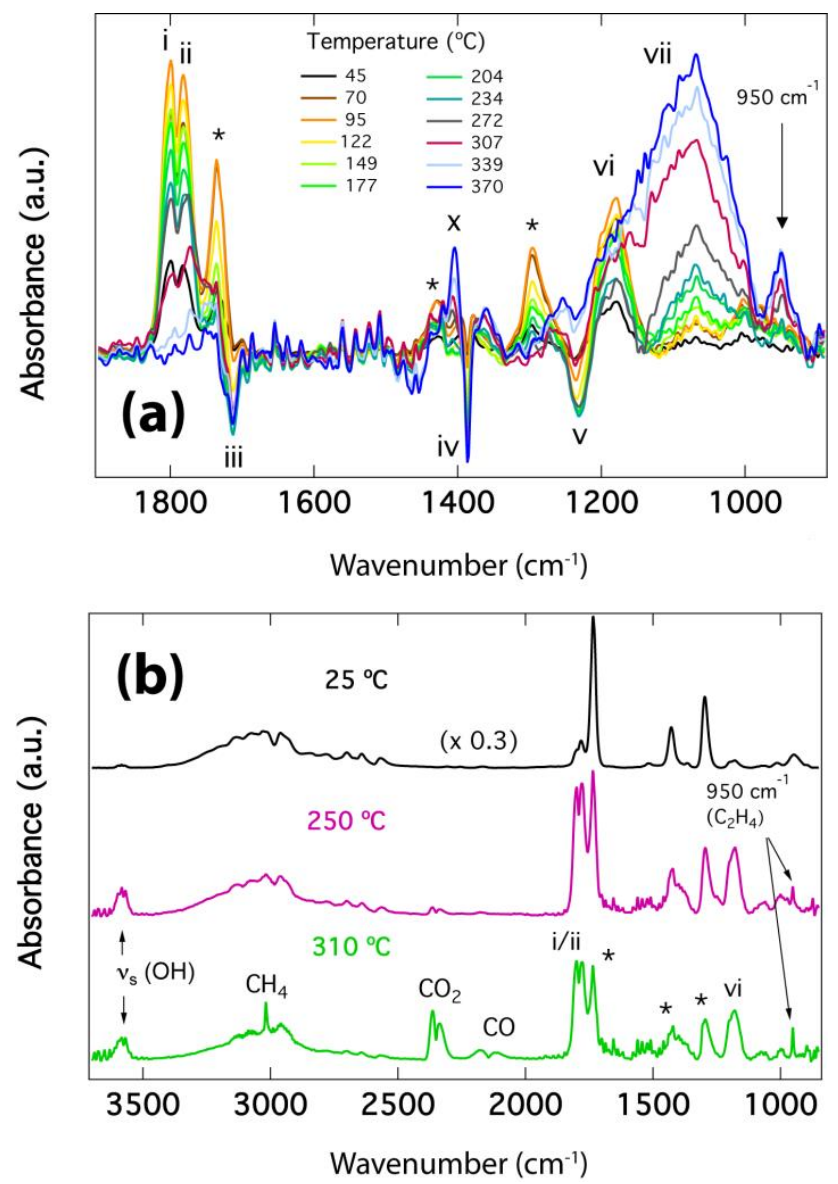

Figure S2. Temperature-dependent DRIFTS spectra for $\mathrm{Mo}_{2} \mathrm{C}$ samples treated with acetic acid and heated in $4 \% \mathrm{H}_{2}$ in Ar. (a) Differential spectra for $\mathrm{Mo}_{2} \mathrm{C}$ sample diluted in $\mathrm{KBr}$. Baseline spectrum was the $\mathrm{D}_{2}$-reduced $\mathrm{Mo}_{2} \mathrm{C}$ sample. Before heating, gas-phase acetic acid was removed by evacuation for several hours. (b) Undiluted $\mathrm{Mo}_{2} \mathrm{C}$ sample heated in $4 \% \mathrm{H}_{2}$ in $\mathrm{Ar}$ with a constant acetic acid overpressure. The baseline for this experiment was the sample after $\mathrm{H}_{2}$ reduction and before acetic acid exposure. In all spectra, peaks corresponding to gas-phase or physisorbed acetic acid are labeled with asterisks and chemisorbed acetic acid adsorbates are labeled $\mathrm{i}-\mathrm{x}$. Gas-phase reaction products are labeled in (b). 


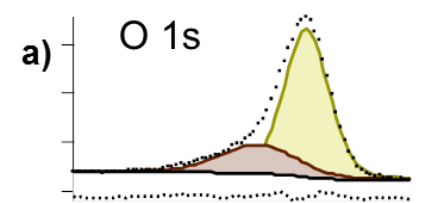

b)

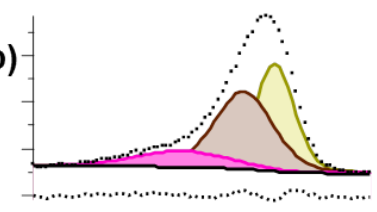

c)

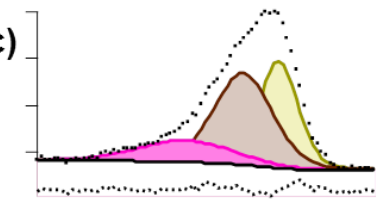

d)
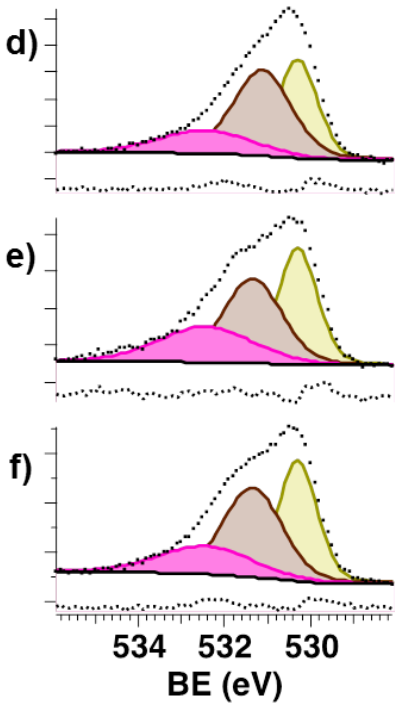
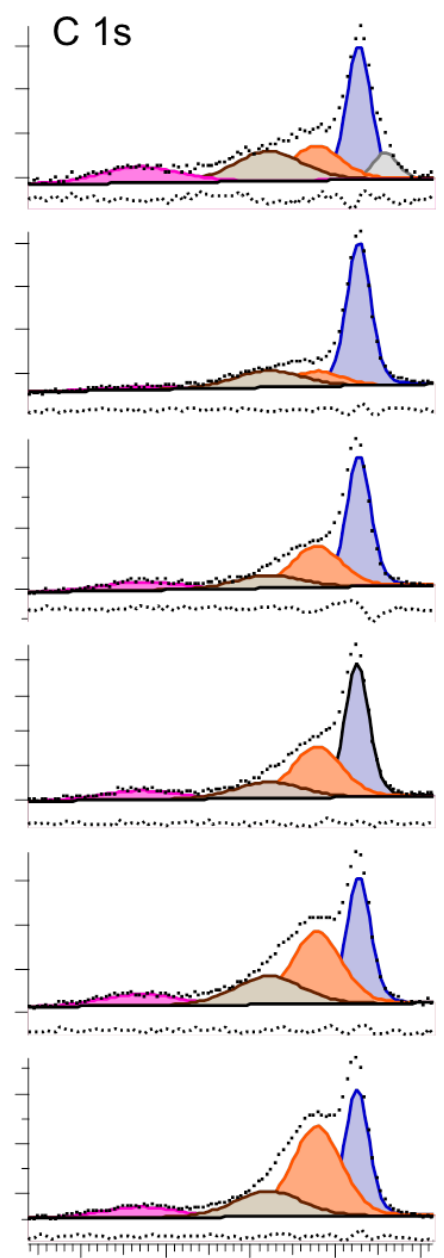

$290 \quad 288 \quad 286 \quad 284 \quad 282$
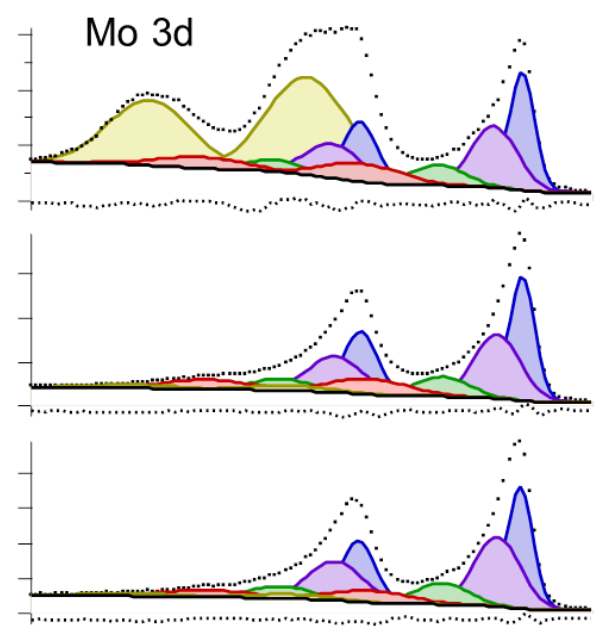

$\because$
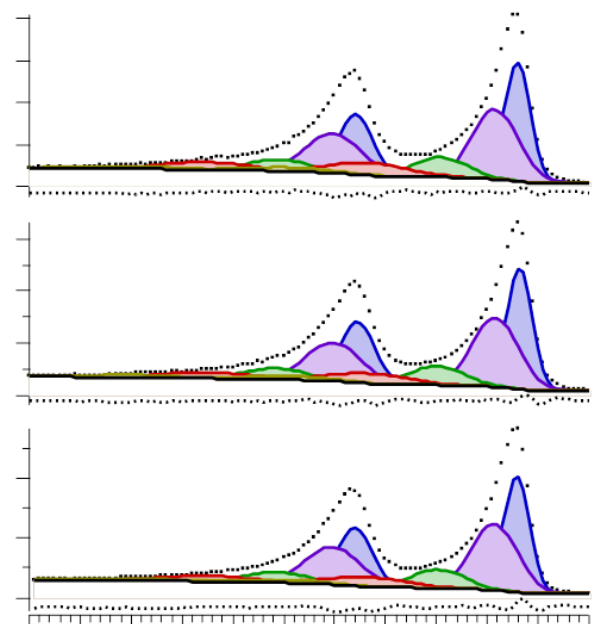

238

$\begin{array}{lllll}236 & 234 & 232 & 230 & 228\end{array}$ $\mathrm{BE}(\mathrm{eV})$

$\mathrm{BE}(\mathrm{eV})$

Figure S3. Core level O 1s, C 1s, and Mo 3d XPS spectra for the $\mathrm{Mo}_{2} \mathrm{C}$ catalyst exposed to various treatments: (a) passivated (as-synthesized), (b) $\mathrm{H}_{2}$ pretreatment at $400{ }^{\circ} \mathrm{C}$, (c) $5 \mathrm{~min}$ TOS, (d) $10 \mathrm{~min}$ TOS, (e) $1 \mathrm{~h}$ TOS, and (f) $2 \mathrm{~h}$ TOS. Dotted line beneath spectra corresponds to the fit residual. See Table S3 for fit parameters. Reaction conditions: $50 \mathrm{mg}$ catalyst, $350{ }^{\circ} \mathrm{C}$, atmospheric pressure, $1.5 \mathrm{~mol} \%$ acetic acid, $11 \mathrm{~mol} \% \mathrm{H}_{2}$, and bal $\mathrm{He}$ (total flow rate of ca. 45.5 $\mathrm{mL} / \mathrm{min})$. 


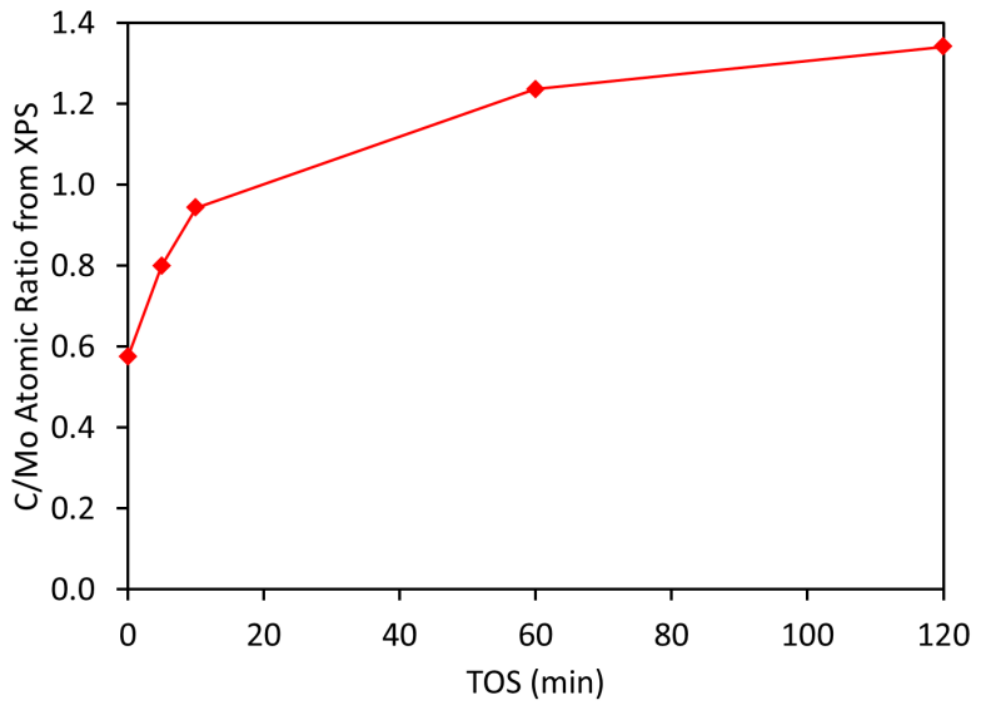

Figure S4. C/Mo atomic ratio (determined by XPS) on the surface of $\mathrm{Mo}_{2} \mathrm{C}$ as a function of TOS. Data point at TOS $=0$ min corresponds to the $\mathrm{Mo}_{2} \mathrm{C}$ catalyst after $\mathrm{H}_{2}$ pretreatment. Reaction conditions: $50 \mathrm{mg}$ catalyst, $350{ }^{\circ} \mathrm{C}$, atmospheric pressure, $1.5 \mathrm{~mol} \%$ acetic acid, 11 $\mathrm{mol} \% \mathrm{H}_{2}$, and bal $\mathrm{He}$ (total flow rate of ca. $45.5 \mathrm{~mL} / \mathrm{min}$ ). 

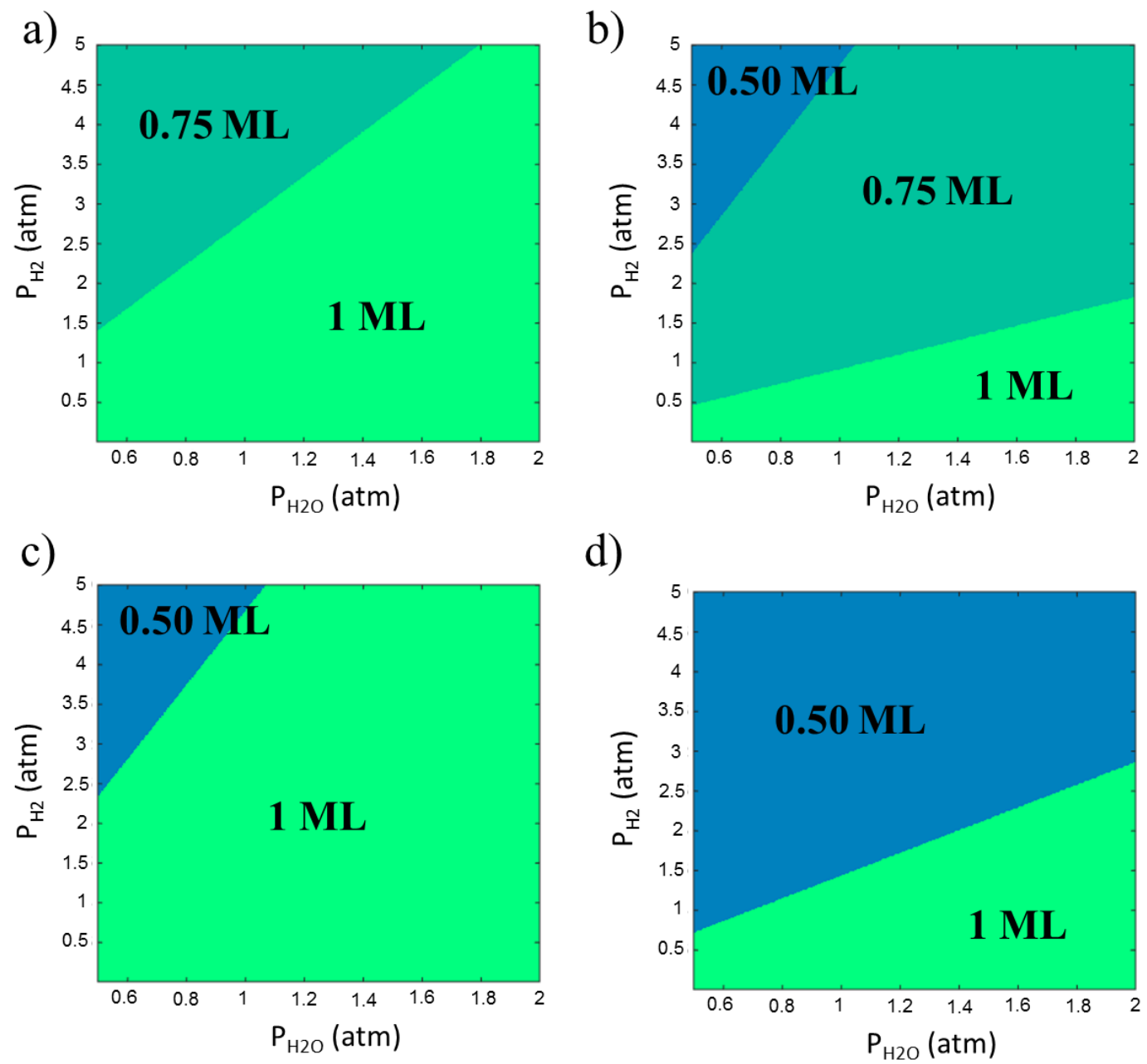

Figure S5. Phase diagrams for atomic oxygen coverage on the (a, b) Mo-terminated $\mathrm{Mo}_{2} \mathrm{C}(001)$ and (c, d) C-terminated $\mathrm{Mo}_{2} \mathrm{C}(001)$ surfaces over a range of typical ex-situ catalytic fast pyrolysis conditions, namely hydrogen and water partial pressures. Figures (a) and (c) were generated at a temperature of $400{ }^{\circ} \mathrm{C}$. Figures (b) and (d) were generated at a temperature of 500 ${ }^{\circ} \mathrm{C}$. $1 \mathrm{ML}$ of oxygen is defined as 1 oxygen atom per each Mo surface atom on the Moterminated surface and 2 oxygen atoms per one $\mathrm{C}$ surface atom on the $\mathrm{C}$-terminated surface. 


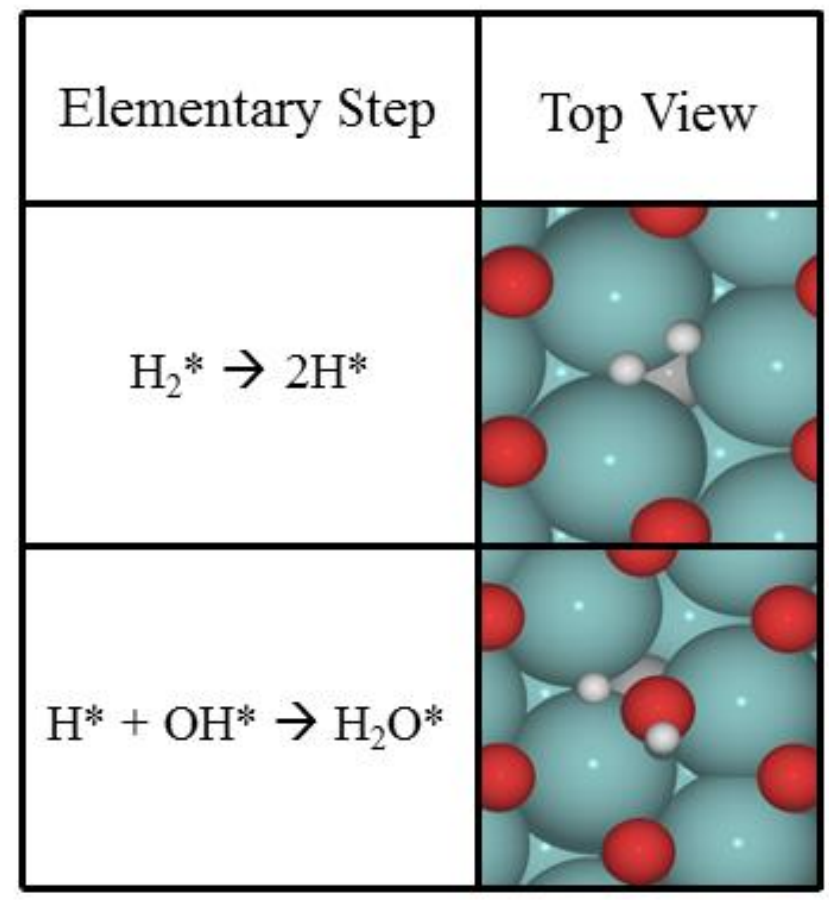

Figure S6. Transition state structures for $\mathrm{H}_{2}$ dissociation at a vacancy site on the $1 \mathrm{ML}$ O/Mo$\mathrm{Mo}_{2} \mathrm{C}(001)$ surface and the transition state structure for water formation on the $1 \mathrm{ML} \mathrm{O} / \mathrm{Mo}-$ $\mathrm{Mo}_{2} \mathrm{C}(001)$ surface. Calculations were performed on a $(2 \times 2)$ unit cell. 


\section{iii. Supporting Tables}

Table S1. Fragmentation patterns and relative mass fragment intensities for reactants and observed products.

\begin{tabular}{|c|c|c|c|c|c|c|c|c|c|c|c|c|c|c|c|c|c|c|c|c|c|c|c|c|}
\hline \multirow{2}{*}{ Compound $^{c}$} & \multicolumn{24}{|c|}{ Mass Fragment ${ }^{a, b}$} \\
\hline & 60 & 58 & 45 & 44 & 43 & 42 & 41 & 39 & 31 & 30 & 29 & 28 & 27 & 26 & 25 & 18 & 17 & 16 & 15 & 14 & 13 & 12 & 4 & 2 \\
\hline Acetic Acid & 44 & - & 83 & - & 100 & - & - & - & 4 & - & 20 & 63 & - & - & - & - & - & - & 34 & 24 & - & - & - & - \\
\hline $\begin{array}{l}\text { Carbon } \\
\text { Dioxide }\end{array}$ & - & - & 1 & 100 & - & - & - & - & - & - & - & 15 & - & - & - & 1 & - & 9 & - & - & - & 3 & - & - \\
\hline Acetone & - & 23 & 1 & 3 & 100 & 7 & 3 & 11 & - & - & 5 & 18 & 7 & 6 & 1 & 2 & - & 1 & 24 & 5 & 1 & - & - & - \\
\hline Ethanol & - & - & 37 & 3 & 9 & 3 & 1 & - & 100 & 6 & 27 & 34 & 29 & 17 & 3 & 4 & 1 & 1 & 9 & 5 & 2 & 1 & - & - \\
\hline Ethane & - & - & - & - & - & - & - & - & - & 26 & 21 & 100 & 33 & 23 & 3 & - & - & - & 4 & 3 & - & - & - & - \\
\hline Acetaldehyde & - & - & 3 & 59 & 33 & 11 & 5 & - & 1 & 2 & 100 & 15 & 5 & 13 & 5 & 2 & 1 & 10 & 66 & 26 & 11 & 5 & - & - \\
\hline $\begin{array}{c}\text { Carbon } \\
\text { Monoxide }\end{array}$ & - & - & - & - & - & - & - & - & - & - & - & 100 & - & - & - & 1 & - & 2 & - & - & - & 3 & - & - \\
\hline Ethylene & - & - & - & - & - & - & - & - & - & - & 2 & 100 & 59 & 58 & 11 & - & - & - & 1 & 4 & 2 & 1 & - & - \\
\hline Water & - & - & - & - & - & - & - & - & - & - & - & - & - & - & - & 100 & 24 & 3 & 1 & - & - & - & - & - \\
\hline Methane & - & - & - & - & - & - & - & - & - & - & - & - & - & - & - & 1 & 2 & 100 & 76 & 13 & 6 & 2 & - & - \\
\hline Helium & - & - & - & - & - & - & - & - & - & - & - & - & - & - & - & - & - & - & - & - & - & - & 100 & - \\
\hline Hydrogen & - & - & - & - & - & - & - & - & - & - & - & - & - & - & - & - & - & - & - & - & - & - & - & 100 \\
\hline 'he mass & & & & & & & & & & & & & & & & & & & & & & & & 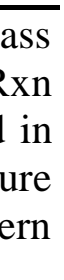 \\
\hline
\end{tabular}

Table S2. Correction factors and primary mass fragments for compounds of interest.

\begin{tabular}{ccc}
\hline Compound & Primary Mass Fragment & Correction Factor \\
\hline Acetic Acid & 45 & 2.9 \\
Carbon Dioxide & 44 & 1.3 \\
Acetone & 43 & 1.7 \\
Ethanol & 31 & 2.1 \\
Ethane & 30 & 1.8 \\
Acetaldehyde & 29 & 2.8 \\
Carbon Monoxide & 28 & 1.0 \\
Ethylene & 26 & 2.1 \\
Water & 18 & 1.2 \\
Methane & 16 & 1.8 \\
Helium & 4 & 0.8 \\
Hydrogen & 2 & 0.6 \\
\hline
\end{tabular}


Table S3. XPS fit parameters.

\begin{tabular}{|c|c|c|c|c|c|c|c|c|c|c|}
\hline \multicolumn{3}{|c|}{ C 1s Model } & \multicolumn{3}{|c|}{ O 1s Model } & \multicolumn{5}{|c|}{ Mo 3d Model } \\
\hline $\begin{array}{c}\text { Peak } \\
\text { Assignment }\end{array}$ & \begin{tabular}{|c|} 
Peak \\
Position \\
$(\mathrm{eV})$
\end{tabular} & $\begin{array}{c}\text { FWHM } \\
(\mathrm{eV})\end{array}$ & $\begin{array}{c}\text { Peak } \\
\text { Assignment }\end{array}$ & \begin{tabular}{|c|} 
Peak \\
Position \\
$(\mathrm{eV})$
\end{tabular} & $\begin{array}{c}\text { FWHM } \\
(\mathrm{eV})\end{array}$ & $\begin{array}{c}\text { Peak } \\
\text { Assigment }\end{array}$ & $\begin{array}{c}5 / 2 \text { Peak } \\
\text { Position } \\
\text { (eV) }\end{array}$ & $\begin{array}{c}\text { FWHM } \\
(\mathrm{eV})\end{array}$ & $\begin{array}{c}\text { Doublet } \\
\text { Separation } \\
(\mathrm{eV}) \\
\end{array}$ & \begin{tabular}{|c|} 
Doublet \\
Broadening \\
$(\mathrm{eV})$
\end{tabular} \\
\hline Carbidic & 283.5 & 0.68 & Mo Oxide & 230.3 & 1.07 & $\begin{array}{c}\mathrm{Mo}^{2+} \\
\left(\mathrm{Mo}_{2} \mathrm{C}\right)\end{array}$ & 228.4 & 0.60 & 3.18 & 0.2 \\
\hline Adventitious & 284.4 & 1.38 & $\begin{array}{c}\text { Mo } \\
\text { Oxycarbide }\end{array}$ & 531.2 & 1.60 & $\mathrm{Mo}^{3+}$ & 228.9 & 1.07 & 3.18 & 0.2 \\
\hline $\begin{array}{c}\text { Oxidative } \\
\text { (C-O) }\end{array}$ & 285.6 & 1.88 & Hydroxyl & 532.5 & 2.59 & $\begin{array}{c}\mathrm{Mo}^{4+} \\
\left(\mathrm{MoO}_{2}\right)\end{array}$ & 229.9 & 1.24 & 3.18 & 0.2 \\
\hline $\begin{array}{l}\text { Oxidative } \\
(\mathrm{C}=\mathrm{O})\end{array}$ & 288.6 & 2.20 & & & & $\mathrm{Mo}^{5+}$ & 231.3 & 1.80 & 3.18 & 0.2 \\
\hline & & & & & & $\begin{array}{c}\mathrm{Mo}^{6+} \\
\left(\mathrm{MoO}_{3}\right)\end{array}$ & 232.9 & 1.90 & 3.11 & 0.0 \\
\hline
\end{tabular}


iv. Supporting References

(1) Ausloos, P.; Clifton, C. L.; Lias, S. G.; Mikaya, A. I.; Stein, S. E.; Tchekhovskoi, D. V.; Sparkman, O. D.; Zaikin, V.; Zhu, D. J. Am. Soc. Mass Spectrom. 1999, 10, 287-299.

(2) Barwick, V.; Langley, J.; Mallet, A.; Stein, B.; Webb, K. Best Practice Guide for Generating Mass Spectra, LGC: London, U.K. 2006.

(3) Lecchi, P.; Zhao, J.; Wiggins, W. S.; Chen, T.-H.; Yip, P. F.; Mansfield, B. C.; Peltier, J. M. J. Am. Soc. Mass Spectrom. 2009, 20, 398-410.

(4) Stein, S. E. Mass Spectra. In NIST Chemistry WebBook;Linstrom, P. J., Mallard, W. G., Eds.; NIST Standard Reference Database Number 69; National Institute of Standards and Technology: Gaithersburg, 2015; http://webbook.nist.gov.

(5) Zhang, Q.; Alfarra, M. R.; Worsnop, D. R.; Allan, J. D.; Coe, H.; Canagaratna, M. R.; Jimenez, J. L. Environ. Sci. Technol. 2005, 39, 4938-4952.

(6) Ko, E. I.; Benziger, J. B.; Madix, R. J. J. Catal. 1980, 62, 264-274. 